\title{
Simple League Championship Algorithm
}

\author{
M. James Stephen \\ Research Scholar, Dept. of CS\&SE, Andhra \\ University, India
}

\author{
Prasad Reddy P.V.G.D \\ Professor, Dept. of CS\&SE, Andhra University, \\ Visakhapatnam, India
}

\begin{abstract}
There is a lot of discussion in the literature on the advantages of one soft computing technique over the other. Number of new meta heuristic algorithms are made available for solving different optimization problems. But always there is some ambiguity in deciding which technique is better than the other to solve a specific optimization problem in a particular context.

A new methodology has been proposed in this paper which provides some theoretical frame work to use multiple optimization techniques at the same time onto a single optimization problem. This new approach has been implemented to solve image enhancement problem in a fingerprint. First the methodology of this new approach has been described then the application of the proposed methodology is presented with some experimental results.
\end{abstract}

\section{General Terms}

Optimization technique, Optimization problem, Simple League Championship Algorithm, Teams, Strategy

\section{Key words}

Multi optimization technique, Transformation function, Fingerprint image enhancement, objective criterion

\section{INTRODUCTION}

There has been a paradigm shift in the computing world from traditional algorithmic hard computing approaches to soft computing techniques. These soft computing approaches have been gaining lot of focus to solve many constrained and unconstrained, linear and non linear problem. The literature in the soft computing reveal that soft computing approaches are performing much better than the traditional approach in solving many optimization techniques.

Researchers on this area of soft computing techniques are coming up with new techniques. Whenever a new technique is introduced, the inventors claim that their techniques are better than other existing optimization techniques. The claim is based on the results of the experiments that are conducted on standard bench mark problems.

After studying many optimization techniques covering various approaches from different variants of particle swarm optimization to latest teaching learning based optimization technique, it is understood that it cannot be decided that one technique is far better than other basing completely on the experimental results on bench mark functions.

Soft computing optimization techniques follow stochastic approach without having a standard mathematical frame work. They operate on randomly selecting the parameter's values. So there is every possibility that some technique might bring out better optimization results than the other on a particular context.

In the present work, many experiments were carried out on a specific problem of image enhancement in a fingerprint image. For image enhancement problem, a transformation function is required. So in the present work three transformation functions (filters) are used. From the experiments it is noted that some technique can perform better than the other on a particular optimization problem of image enhancement in a specific context. This gave motivation to develop this new technique, called 'Simple League Championship Algorithm' (SLCA). The proposed methodology is explained with fingerprint image enhancement context. But it can be extended to solve any optimization problem.

\subsection{Diversification and Intensification}

In reviewing any Meta heuristic algorithms, two major components are focused. They are diversification and intensification. These two components are seemingly contradicting each other, but their balanced combination is crucially important to the success of any meta-heuristic algorithms [4], [5], [6].

Proper diversification or exploration makes sure the search in the parameter space can explore as many locations and regions as possible in an efficient and effective manner. It also ensures that the evolving system will not be trapped in biased local optima. Diversification is often represented in the implementation as the randomization and/or additional stochastic component superposed onto the deterministic components. If the diversification is too strong, it may explore more accessible search space in a stochastic manner, and subsequently will slow down the convergence of the algorithm. If the diversification is too weak, there is a risk that the parameter space explored is so limited and the solutions are biased and trapped in local optima, or even lead to meaningless solutions.

On the other hand, the appropriate intensification or exploitation intends to exploit the history and experience of the search process. It aims to ensure to speed up the convergence when necessary by reducing the randomness and limiting diversification.

If the intensification is too strong, it could result in premature convergence, leading to biased local optima or even meaningless solutions, as the search space is not well explored. If the intensification is too weak, convergence becomes slow.

The optimal balance of diversification and intensification is required, and such a balance itself is an optimization process. Fine-tuning of parameters is often required to improve the efficiency of the algorithms for a particular problem. No Free 
Lunch Theorems for Optimization [7] presents a framework to explore the connection between effective optimization algorithms and the problems they are solving. A substantial amount of studies might be required to choose the right algorithms for the right optimization problems [8], though it lacks a systematic guidance for such choices.

\section{Proposed Methodology}

The league championship algorithm (LCA) is a novel Meta heuristic algorithm designed based on the metaphor of sporting competitions in sport leagues. The complete details of League Championship Algorithm can found in [2]

Basing on the concept presented in the original LCA, a simple concept, which is called a 'Simple LCA' (SLCA) is designed to suit the requirement of using different transformation functions and different optimization techniques in various combinations for the purpose of solving an optimization problem. The other details in the original LCA are ignored to make the complex algorithm simple.

For implementing the SLCA some idealized rules have been formed. Each of the following idealized rules has been considered for making different steps in the proposed SLCA algorithm.

Idealized rule 1: It is more likely that a team with better playing strength wins the game. The term "playing strength" refers to ability of performing better than the team.

Idealized rule 2: The outcome of a game is not predictable, in spite of knowing the teams' playing strength. In other words, it is not unlikely that the Australian cricket team losing to Kenya.

Idealized rule 3: The probability that team $\mathrm{i}$ beats team $\mathrm{j}$ is assumed to be equal from both team's point of view.

Idealized rule 4: The outcome of the game is only win or loss; there is no tie.

Idealized rule 5: When team $i$ beats team $j$, it is considered that team $\mathrm{i}$ has adopted better strategy than team $\mathrm{j}$.

Idealized rule 6: Teams only focus on their upcoming match in the next round without regards of the other future matches. Given the past results, new strategy will be adopted for the next match.

Idealized rule 7: The winning team continues with the same strategy which was adopted in the last game for the next match. The loosing team changes their strategy for the next game..

\subsection{Simple League Championship Algorithm for Fingerprint image Enhancement}

Here image enhancement is the optimization problem and different optimization techniques are used to solve it.

Three transformation functions and three optimization techniques are used with different combination using SLC Algorithm. This new methodology is described with fingerprint image enhancement problem.

\subsection{Transformation Functions}

The following three transformation functions are used in the present chapter for the purpose of fingerprint image enhancement.

Transformation Function-1: The $1^{\text {st }}$ transformation function, which is used in the present proposed methodology, is

$$
g(i, j)=\left[\frac{(d * G)}{\sigma(i, j)+\mathrm{b}}\right][f(i, j)-c * m(i, j)]+m(i, j) a
$$

Transformation Function-2: The $2^{\text {nd }}$ transformation function considered in this proposed methodology is

$$
\begin{aligned}
& g(i, j)=d *\left(\left(\sigma_{\eta}^{2}(i, j) / \sigma_{\mathrm{L}}^{2}(i, j)+b\right)\right)[f(i, j)-c * m(i, j)]+ \\
& m(i, j) a
\end{aligned}
$$

Transformation Function-3: The third transformation function that is designed for this proposed methodology is

$$
\begin{aligned}
& g(i, j)=f(i, j)-d *\left(\left(\sigma_{\eta}^{2}(i, j) / \sigma_{\mathrm{L}}^{2}(i, j)+b\right)\right)[f(i, j)-c * \\
& m(i, j)]+m(i, j) a
\end{aligned}
$$

Where $G$ is the global mean, $m$ is the local mean, $\sigma(i, j)$ is the standard deviation, $\sigma_{\eta}^{2}(i, j)$ is the global variance, $\sigma_{\mathrm{L}}^{2}(i, j)$ is the local variance. $[a, b, c, d]$ are the random variables in the range $[0,1]$.

\subsection{Optimization Techniques}

The following three optimization Techniques are used in the proposed methodology.

1. Teaching Learning Based Optimization

2. Particle Swarm Optimization

3. Harmonic Search

\subsection{Teams \& Strategy}

Here Each Transformation Technique is considered as one team and each optimization technique is considered as the team's strategy of playing in the match. So here the transformation functions as teams and optimization techniques as teams' strategy are defined as follows

Team 1 : Transformation Function (1)

Strategy : Modified Teaching Learning Based Optimization (M-TLBO)

Team2 : Transformation Function (2)

Strategy : Particle Swarm Optimization (PSO)

Team3 : Transformation Function (3)

Strategy : Modified Harmonic Search (M-HS)

Initially three teams with their own strategy plays according to a tournament structure (in the present work every team plays in three rounds). Here Transformation function is a team, $[a, b, c, d]$ are the randomly played shots according to a specific strategy (optimization technique), which is adopted by the team. The winning team will not change the strategy; whereas the loosing team changes the strategy for the next match.

Here playing implies 'taking an input fingerprint image and enhancing it'. Every team is given the same input fingerprint image and same objective criterion is used to evaluate the rate of enhancement. Entropy and Peak Signal-to-Noise ratio are used as evaluation criterion.

\subsection{Winner/loser recognition}

In the original LCA algorithm, the methodology of recognizing winner / looser is also done randomly. It is not appealing because it is as similar to deciding the winner / looser based on tossing the coin after playing the match completely. 
Here in this work, winner / looser is recognized based on the objective criterion. At every iteration, each team takes the same input image and the transformation function is applied with the randomly generated [a,b,c,d] values according the optimization technique to produce an enhanced fingerprint image. The fitness value based on the objective criterion is calculated, which is nothing but the score. Whichever team gets the highest score is declared as winner.

\subsection{Changing the strategy}

When a team loses in a match, it is the responsibility of the coach to think about changing the strategy of playing. Here it is changing the optimization technique. When a specific transformation function (specific team) had got lower score with a specific optimization technique (Strategy) than other team, then the same transformation function is used with different optimization technique (strategy) in the next match.

Finally the best Team with the best strategy is selected based on the objective criterion (fitness value) as the champion and the corresponding best enhanced fingerprint image is returned.

\subsection{SLC Algorithm}

Step 1: Initialize each team with a specific strategy

Step 2: Play the matches at first round (Here it is a three round format)

Step 3: Decide the winner of the first round based on the objective criterion

Step 4: Winner / Looser will go for the next match at next round by adopting the strategy as follows

- Winning team will not change the strategy for next match

- Losing team will take up a new strategy for the next match

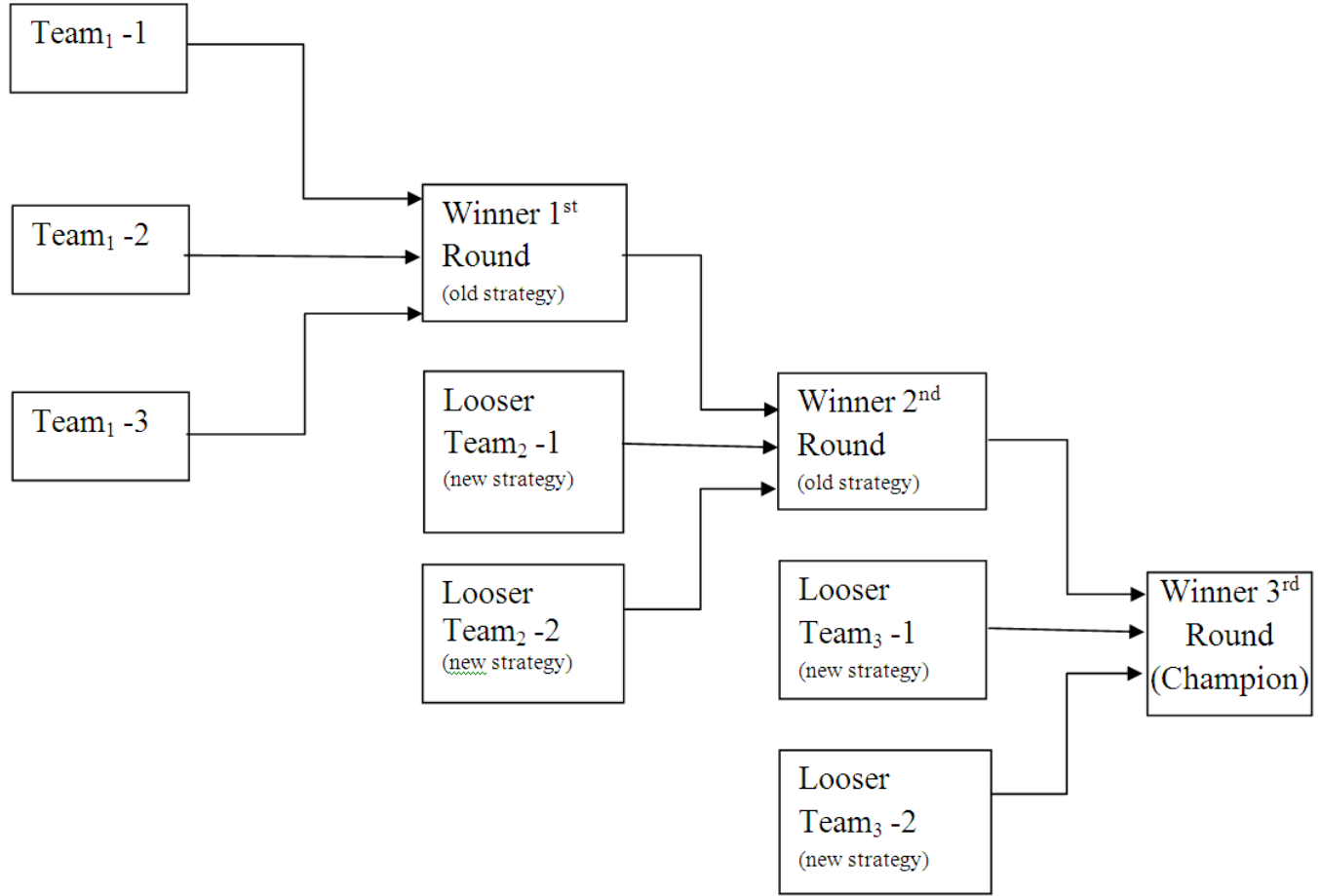

Figure 1: Flow graph of League Championship Algorithm (preferably the one that is not used so far)

Step 5: Continue playing by repeating step-4 till the tournament schedule is complete (Here only three rounds)

Step 6: Decide the winner based on the highest score with respect to the evaluation criteria.

Both fitness function, Fit (X) with Entropy and Peak Signal to Noise Ratio (PSNR) are used as multi-objective criterion [9]

The process of implementation of the proposed methodology can be better understood through the flow graph, presented in Figure 1.

The fingerprint image enhancement problem is solved by different optimization techniques independently first. The authors presented fingerprint image enhancement with Particle Swarm Optimization (PSO) in [9]. The other methodologies of enhancing fingerprint image enhancement using Modified Teaching Learning Based Optimization (MTLBO) and Modified Harmony Search (M-HS) were also implemented and communicated the results to different journals.

Enormous experiments are conducted on huge fingerprint dataset. Comparing the results of enhancement process with different optimization technique, M-TLBO performed better with Transformation function-2, but it is observed that other combinations performed better for some particular fingerprint images. 


\section{Experimental Results}

Experiments were carried out on ATVS-Fake Fingerprint Database (ATVS-FFp DB) and FVC 2002 fingerprint database. "ATVS-Fake Fingerprint Database (ATVS-FFp DB)" is one of the bench marked databases that are made publicly available at BIT website. In the present work this particular database is used for various experiments. The fake and the real / original portions of the databases are used. The creation of this fingerprint database is described in [3]. The fake fingerprint database contains the poorest quality fingerprints. The proposed SLCA enhancement technique is applied on such poor quality fake fingerprints to improve their quality. Many experiments were also carried out on fingerprint dataset, collected from FVC 2002 of MSU [10]

The third open source package of NBIS [1], NFIQ, is a fingerprint image quality algorithm. It takes a fingerprint image and analyzes the overall quality of the image returning an image quality number ranging from 1 for highest to 5 for lowest. In the present work this package is used to validate quality of fingerprint images before and after the enhancement through proposed methodology. Results were presented only for 32 sample fingerprints. The same sample set is also used to present results in PSO based enhancement [9].

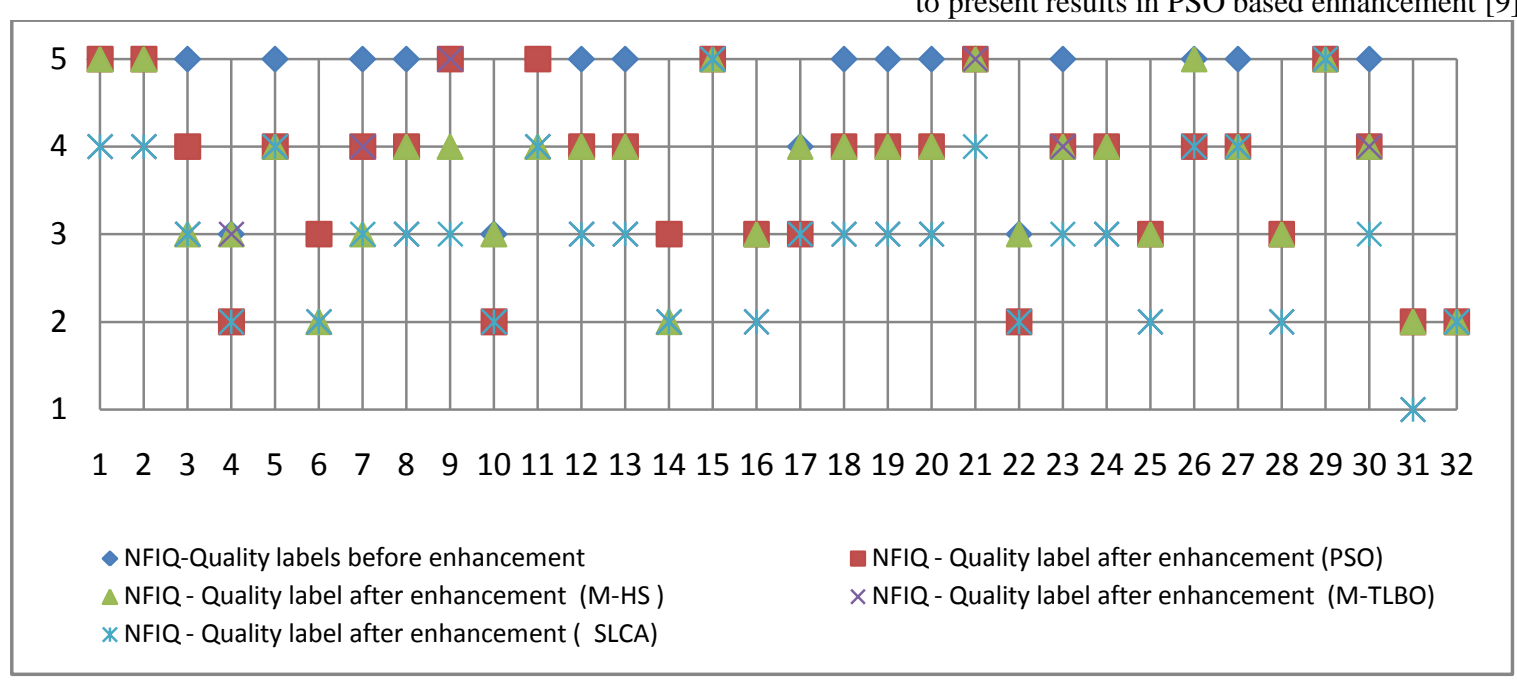

Figure 2: Comparative Results of NFIQ Quality Labels- before and after enhancement through M-TLBO, PSO, M-HS \& SLCA

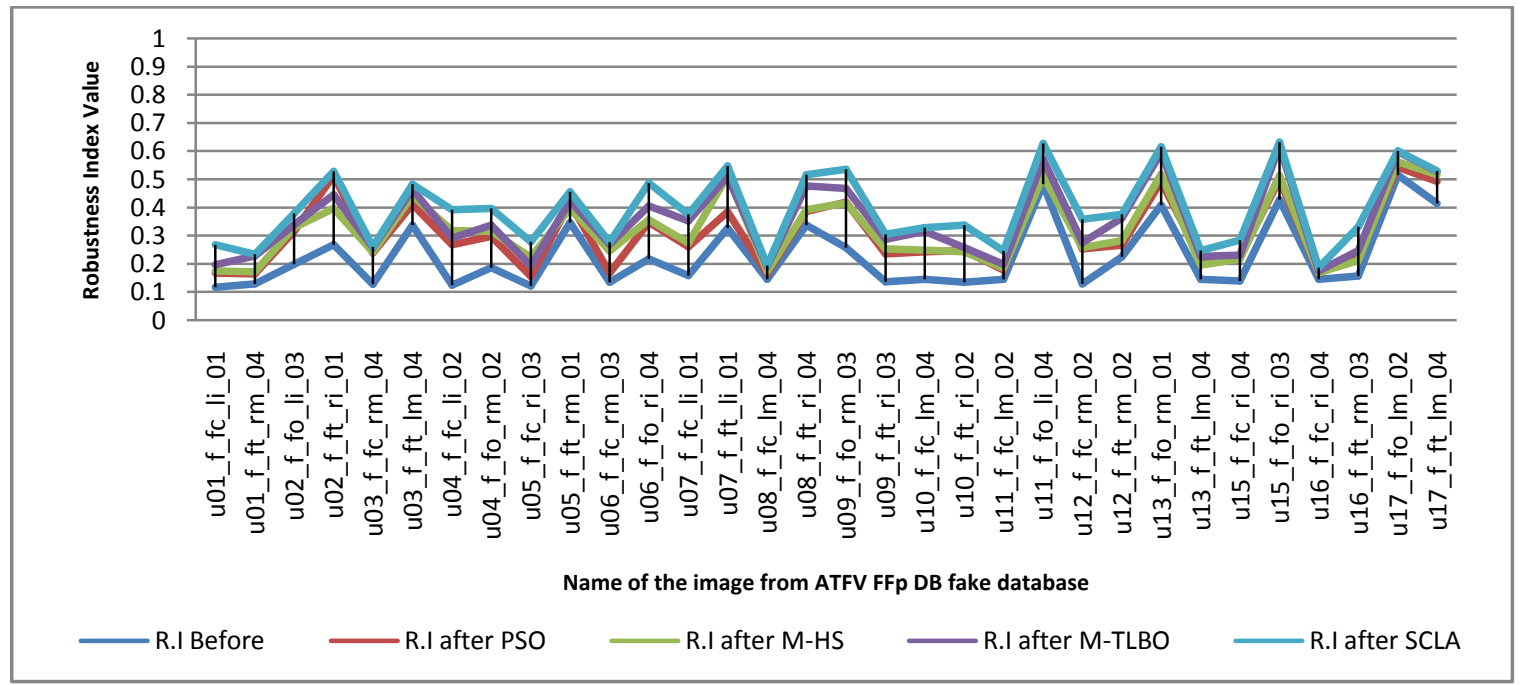

Figure 3: Comparative results of Robustness Index: - before and after enhancement with PSO, M-TLBO, M-HS \& SLCA

The graph in figure 2 shows quality labels (NFIQ) of fingerprints before and after enhancement. The quality scores of the new methodology using all the three transformation functions and three optimization techniques in various combinations with the help of Simple League Championship Algorithm as well as the scores with the application of individual optimization techniques / transformation functions are presented. It can be observed from the resulted data that the proposed new methodology always performs better than the other techniques that are used independently.
Some sample comparative results with respect to the Robustness Index of Proposed Enhancement Technique with SLCA and other enhancement techniques independently are presented in figure 3 . The results are presented for the same sample fingerprint data set as in [9] for better result analysis.

It can be observed from the graph in figure 4 that the Robustness Index that is obtained through the proposed SLCA technique is always higher than the other techniques. So it can be concluded that the proposed methodology of fingerprint 
image enhancement with SLCA works better than the various techniques independently. These results establish the efficacy of the newly proposed method of using multiple optimization techniques to solve a single optimization problem.

The experimental findings with respect to the verification performance on the standard fingerprint dataset, collected from FVC 2002 of MSU [10] are presented in figure 4. The proposed enhancement technique is evaluated on a set of 800 images (100 fingers, 8 images each from DB3_A) derived from FVC2002. The total number of genuine and impostor comparisons are 2800 and 4950 respectively. Two tests are carried out. In the first test, verification is performed on the fingerprints before applying enhancement and in the second test verification is done on the fingerprints after enhancement. ROC graph can be seen in figure 4, where genuine acceptance rate is plotted against the false acceptance rate at different operating points.

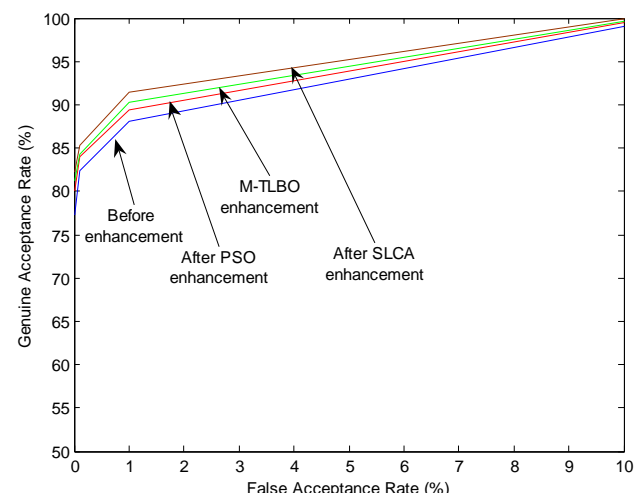

Figure 4: ROC curves before and after SLCA \& M-TLBO enhancement on FVC 2002 DB

From these experimental results, it can be observed that the performance of the verification system is further improved with the proposed image enhancement technique. The results of PSO based enhancement and M-TLBO enhancement were also presented to show the superiority of the proposed technique over the other. It can be noted that at this stage, image enhancement is done basically to remove noise from the fingerprint image but other typical fingerprint filtering techniques have not been applied. As such, the results obtained demonstrate that verification performance can be increased by effectively eliminating the noise from fingerprint images.

\section{CONCLUSION}

A new approach of using multiple optimization techniques on a single optimization problem is proposed in this paper. The Simple League Championship Algorithm (SLCA) is proved to serve this purpose of using multiple optimization techniques on a single optimization problem. Based on this new concept a new methodology of fingerprint image Enhancement using Multiple Transformation Functions and Multiple Optimization Techniques through SLCA has been proposed and experimented. The results are encouraging and that provides the motivation for further exploration. There is a lot of scope for improvement in the proposed methodology. Changing the tournament structure may give more scope for exploring more possibilities of experimenting with different combination of transformation functions and optimization techniques. There is no need that the total number of teams and strategies must be equal. The size of the teams and strategies can be increased for much better results. But there is a tradeoff between the desire for more optimization and computational burden / design complexity. After completing the proposed enhancement technique, the other typical fingerprint filtering techniques can be applied for further improvement of fingerprint's quality.

\section{REFERENCES}

[1] Watson, G. I., Garris, M. D., et al. (2004). User's guide to NIST fingerprint image software 2 (NFIS2) / (NBIS) National Institute of Standards and Technology.

[2] Ali Husseinzadeh Kashan, An efficient algorithm for constrained global optimization and application to mechanical engineering design: League championship algorithm (LCA), Computer-Aided Design 43 (2011) 1769-1792.

[3] J. Galbally, J. Fierrez, F. Alonso-Fernandez and M. Martinez-Diaz, "Evaluation of Direct Attacks to Fingerprint Verification Systems",Telecommunication Systems, Special Issue on Biometrics, Vol. 47, n. 3, pp. 243-254, January 2011.

[4] Yang XS (2008) Nature-inspired Metaheuristic Algorithms. Luniver Press.

[5] Blum C and Roli A (2003) Metaheuristics in combinatorial optimization: Overview and conceptural comparison. ACM Comput. Surv., 35:268-308.

[6] Dorigo M and Blum C (2005) Ant colony optimization theory: A survey. Theor. Comput. Sci., 344:243-278.

[7] Wolpert DH and Macready WG (1997). No free lunch theorems for optimization. IEEE Transaction on Evolutionary Computation, 1:67-82.

[8] Yang XS (2008) Mathematical Optimization: From Linear Programming to Metaheuristics, Cambridge Int. Science Publishing, UK.

[9] James M Stephen, Prasad Reddy PVGD, Article: Fingerprint Image Enhancement through Particle Swarm Optimization. International Journal of Computer Applications 66(21):34-40, March 2013.

[10] D. Maio, D. Maltoni, R. Cappelli, J.L. Wayman and A.K. Jain, "FVC2002: Second Fingerprint Verification Competition", in proceedings 16th International Conference on Pattern Recognition, Québec City, vol.3, pp.811-814, 